\title{
El retroceso de la dependencia en pro de la estabilidad presupuestaria y el fomento de la competitividad ${ }^{1}$
}

\section{Manuela Durán Bernardino}

Departamento de Derecho del Trabajo y de la Seguridad Social, Universidad de Granada <mduranb@ugr.es>

\begin{abstract}
Azken urteotan hainbat erreforma onartu dira mendekotasunaren alorrean, aurrekontuaren egonkortasuna bermatzeko eta lehiakortasuna sustatzeko helburuarekin. Erreforma horiek atzerakada nabarmena dira mendekotasun-sistema babesteko zereginean, Gizarte Segurantzatik kanpo uzten dituelako herritar ez profesionalak, Estatuko Administrazio Nagusiaren ekarpena murrizten duelako edo Mendekotasunaren Legearen aplikazio progresiboaren egutegia geldiarazten duelako. Dagoeneko zortzi urte dira indarrean jarri zela Mendekotasunaren Egoeran dauden Pertsonen Autonomia eta horien Arreta Sustatzeko 39/2006 Legea, abenduaren 14koa, eta artikulu honen bitartez aztertu nahi da, ikuspegi juridiko-kritikotik, benetan zer eragin izan duten Autonomiarako eta Mendekotasunaren Arretarako Sisteman hartutako azken murrizketa-neurriek, eta ebaluatu nahi da zer ondorio izan dituen mendekotasun egoeran dauden milioi batetik gora herritarrengan.
\end{abstract}

\section{GAKO-HITZAK:}

Norberaren autonomia, mendekotasuna, aurrekontumurrizketak, erreformak, babesgabetasuna, iraunkortasuna.
En los últimos años se han adoptado un conjunto de reformas en materia de dependencia con el fin de garantizar la estabilidad presupuestaria y fomentar la competitividad. Dichas reformas originan un notorio retroceso en la acción protectora del sistema de dependencia, al expulsar a los cuidadores no profesionales de la Seguridad Social, reducir la aportación de la Administración General del Estado o paralizar el calendario de aplicación progresiva de la Ley de Dependencia. Tras ocho años de la entrada en vigor de la Ley 39/2006, de 14 de diciembre, de Promoción de la Autonomía Personal y Atención a las Personas en Situación de Dependencia, la finalidad de este artículo es examinar, desde una perspectiva jurídico-crítica, la incidencia real de las últimas medidas de recorte adoptadas en el Sistema para la Autonomía y Atención a la Dependencia, y evaluar sus consecuencias para más de un millón de personas en situación de dependencia.

\section{Palabras Clave:}

Autonomía personal, dependencia, recortes presupuestarios, reformas, desprotección, sostenibilidad.

${ }^{1}$ Una versión previa de este trabajo se presentó en el V Congreso de la Red Española de Política Social (REPS) 'Desigualdad y democracia: política públicas e innovación social', celebrado el 5 y 6 de febrero de 2015 en Barcelona. 


\section{Planteamiento general}

El Estado trata de dar respuesta a la intensa y creciente demanda de protección de las personas en situación de dependencia con la aprobación de la Ley 39/2006, de 14 de Diciembre, de Promoción de la Autonomía Personal y Atención a las Personas en Situación de Dependencia, que ya en su exposición de motivos advierte de la magnitud del problema social que se acota con el concepto de dependencia: "la atención a las personas en situación de dependencia constituye uno de los principales retos de la política social de los países desarrollados". Partiendo de la noción utilizada por el Consejo de Europa, el reto consiste en atender las necesidades de aquellas "personas que, por razones ligadas a la falta o a la pérdida de autonomía física, psíquica o intelectual, tienen necesidad de asistencia y/o ayuda importantes a fin de realizar las actividades corrientes de la vida diaria" (Recomendación no 98, 9, del Comité de Ministros a los Estados miembros relativa a la dependencia).

Así, estamos ante una ley que representa un importante avance de nuestro sistema de protección social, al regular las condiciones básicas dirigidas a garantizar la igualdad en la atención a las personas dependientes, sobre la base del reconocimiento de un derecho subjetivo y universal (Beltrán Aguirre, 2008: 4). Con la aprobación de esta ley, el Estado sienta los cimientos para la configuración del nuevo Sistema para la Autonomía y Atención a la Dependencia (SAAD) que, lejos de integrarse en el sistema de Seguridad Social, se concibe como un sistema de protección específico y autónomo, llamado a ser el cuarto pilar del Estado del bienestar. Debe destacarse desde el principio la enorme complejidad del modelo legal que se pretende instaurar, en el cual están involucrados todos los niveles de las administraciones públicas -Administración General del Estado, administraciones autonómicas, administraciones locales-, el ámbito privado o familiar, las organizaciones de mayores y personas con discapacidad, y las entidades del tercer sector, que igualmente intervienen en el sistema.

Pese a lo positivo de la puesta en marcha de este sistema, lo cierto es que actualmente no podemos hablar de un modelo de protección cuyo desarrollo e implantación efectiva haya culminado. Las reformas emprendidas a consecuencia de la crisis económica han tenido una grave incidencia en la protección dispensada por el SAAD, ya que las medidas aprobadas en 2012 no sólo han provocado la paralización del sistema, sino que su implantación está derivando en un paulatino retroceso de su acción protectora en los términos que en los siguientes epígrafes se analiza.

\section{Cambio de dirección en la configuración del Sistema para la Autonomía y Atención a la Dependencia. Medidas adoptadas}

Con el objeto de garantizar la sostenibilidad presente y futura del sistema y reducir el gasto público, recientemente se han aprobado un conjunto de disposiciones de enorme interés y transcendencia para el estudio técnico-jurídico de la dependencia y para la comprensión del cambio de dirección que se pretende adoptar en la configuración del sistema español de protección social de la dependencia. Se hace aquí referencia al Real Decreto Ley 20/2012, de 13 de julio, de Medidas para Garantizar la Estabilidad Presupuestaria y de Fomento de la Competitividad, y al Acuerdo del Consejo Territorial de 10 de julio de 2012, para la mejora del SAAD, cuya aprobación -junto a las leyes 2/2012, de 29 de junio; 17/2012, de 27 de diciembre; $22 / 2013$, de 23 de diciembre; $y$ $36 / 2014$, de 26 de diciembre- ha supuesto una notoria modificación del originario sistema de dependencia. Para comprenderlas mejor, las medidas aprobadas e implementadas se analizarán por bloques temáticos.

\subsection{Restricción de la prestación económica para cuidados en el entorno familiar}

Con la intención de fomentar las prestaciones de servicio y reducir el gasto público, se adoptan un conjunto de medidas que restringen sumamente la concesión de la prestación económica para cuidados en el entorno familiar. En primer lugar, se introduce un nuevo criterio de asignación del nivel mínimo de protección. Así, la distribución entre las comunidades autónomas del nivel mínimo de protección trasferido por la Administración General del Estado ya no sólo se efectuará conforme al número de beneficiarios y la cuantía por grado de dependencia reconocida para cada uno de ellos ${ }^{2}$, sino que se pondere positivamente la prestación de servicios respecto a la prestación económica por cuidados en el entorno familiar ${ }^{3}$ (propuesta de mejora 15 ${ }^{\mathrm{a}}$, recogida en el Real Decreto Ley y aprobada por el Real Decreto 1050/2013, de 27 de diciembre).

En segundo lugar, el reconocimiento de esta prestación estará sujeto a un plazo suspensivo máximo de dos años para las personas que a 16 de julio de 2012, fecha de entrada en vigor del Real Decreto Ley, no hubieran comenzado a percibir todavía la prestación económica reconocida a su favor (propuesta de mejora $13^{\underline{a}}$ del Acuerdo y disposición adicional séptima del Real Decreto).

Este criterio permitió que, desde que entrara en vigor la Ley de Dependencia, las comunidades autónomas recibiesen una financiación adicional de la Administración General del Estado (AGE), al no diferenciar las prestaciones en función de su existencia previa a la Ley. Es decir, la AGE no sólo contribuía al gasto 'nuevo', generado para cumplir los objetivos de la Ley, sino también al gasto 'consolidado' en las diferentes comunidades autónomas (Ministerio de Sanidad, Política Social e Igualdad, 2011: 188).

${ }^{3}$ No obstante, para evitar desequilibrios demasiado pronunciados y acelerados en la financiación de unas comunidades (las que han venido dando prioridad a las prestaciones de servicios, como es el caso de Castilla y León o Andalucía) frente a otras (en las que tienen un mayor peso las prestaciones económicas, como sucede en las Islas Baleares o Murcia), se prevé que el reparto del nivel mínimo evolucione progresivamente a lo largo de cinco años, con una implantación progresiva de la distribución por beneficiarios y prestaciones. 
Además, en el marco de los recortes y las limitaciones de las prestaciones económicas de cuidado familiar, a partir de la entrada en vigor del Real Decreto Ley, el convenio especial regulado en el Real Decreto $615 / 2007$, de 11 de mayo, pasa a tener carácter voluntario para los cuidadores no profesionales, pudiendo ser suscrito entre el cuidador no profesional y la Tesorería General de la Seguridad Social. Estos convenios surtirán efecto desde la fecha de la solicitud de suscripción del convenio especial (disposición adicional octava). Aquellos convenios especiales que existieran con carácter previo a la entrada en vigor del Real Decreto 20/2012, fueron extinguidos el 31 de agosto de 2012, salvo en aquellos casos en los que el cuidador hubiera solicitado expresamente su mantenimiento con anterioridad al 2 de noviembre de 2012, en cuyo caso se entenderá que subsiste desde el 1 de septiembre de 2012 (disposición transitoria decimotercera).

En cuarto lugar, las cotizaciones a la Seguridad Social por el convenio especial no seguirán a cargo de la Administración General de la Seguridad Social, sino que en aquellos casos en los que el cuidador, de forma voluntaria, decida mantener dicho convenio, se establece con carácter transitorio que, desde el 1 de septiembre hasta el 31 de diciembre de 2012, la cotización a la Seguridad Social tendrá una reducción del $10 \%$ en la cuota total, siendo a cargo de la Administración General del Estado el $5 \%$ de ésta y el $85 \%$ restante, a cargo del cuidador no profesional (disposición transitoria decimotercera). Una vez transcurrido ese periodo, es decir, a partir del 1 de enero de 2013, el Gobierno dejó de cotizar a la Seguridad Social por aquellas personas que cuidaban a un familiar, de forma que el convenio especial pasó a estar a cargo exclusivo del cuidador no profesional (disposición adicional octava y disposición transitoria decimotercera).

Como era de esperar, el número de convenios especiales de cuidadores no profesionales de personas en situación de dependencia se ha reducido drásticamente y de forma inmediata 4 . A de 31 de marzo de 2013 -tan sólo tres meses después-, el número total de convenios suscritos por los cuidadores no profesionales era de 21.685 , correspondientes a 20.993 convenios obligatorios y, tan sólo a 682 convenios voluntarios (Imserso, 2013). Dos años después de las reformas adoptadas (julio 2012), se puede comprobar que el número de cuidadores no profesionales dados de alta ha continuado descendiendo. Si comparamos con los datos de afiliación previos a la reforma, comprobamos que se ha reducido un $91,5 \%$, pasando de 172.363 personas afiliadas antes de la reforma a tan sólo 14.594 en julio de 2014 .

${ }^{4}$ El punto álgido de afiliación de cuidadores no profesionales se alcanzó en julio de 2012 y fue de 180.021 . A partir de dicha fecha y como consecuencia directa del efecto del Real Decreto 20/2012 en el sistema de Seguridad Social, los datos de afiliación han ido cayendo. Tan sólo en los meses de noviembre y diciembre se redujeron en 148.672 (Ministerio de Empleo y Seguridad Social, s/d).
Finalmente, se reducen en un $15 \%$ las cuantías económicas máximas recibidas por los cuidadores familiares (disposición transitoria décima del Real Decreto Ley 20/2012, de 13 de julio, a la que se remite igualmente el Real Decreto 1051/2013, de 27 de diciembre), que podrá ser aumentado por las comunidades autónomas en función de sus necesidades de financiación y presupuestarias (propuesta de mejora $15^{\underline{a}}$ del Acuerdo de 10 de julio) ${ }^{5}$.

\subsection{Reducción en la aportación de la Administración General del Estado a la financiación del sistema de dependencia}

Con la finalidad de generar un ahorro en el gasto de la Administración General del Estado, se adoptan un conjunto de medidas de carácter económico que inciden directamente en los niveles de financiación del sistema de dependencia, redefiniéndose la participación de los diferentes agentes implicados (Administración General del Estado, comunidad autónoma, beneficiario). Según se dispone, la pretensión de estas medidas no es otra que la de alcanzar un "reequilibrio sostenible del Sistema", que garantice el derecho a la promoción de la autonomía personal y la atención a la situación de dependencia.

\subsubsection{Reducción de las cuantías del nivel mínimo de financiación del sistema de dependencia.}

Como se ha visto, el nivel mínimo de protección garantizado a cada beneficiario fue, en 2007 (el primer ejercicio en que se ofrecía la prestación), de 250 euros para personas con gran dependencia de nivel 2 y de 170 euros para personas con gran dependencia de nivel 1 (art 3.3 y anexo al Real Decreto 614/2007). Dichas cuantías debían actualizarse anualmente mediante real decreto, en función de la actualización aplicada al indicador público de renta de efectos múltiples (IPREM) [art. 3.4 del Real Decreto 614/2007, actualmente derogado]. Sin embargo, tan sólo se cumplió con dicha previsión durante los tres ejercicios siguientes ${ }^{6}$.

${ }_{5}^{5}$ Para los beneficiarios que tuvieran reconocido un grado y nivel de dependencia a la entrada en vigor del Real Decreto 20/2012, de 13 de julio, la cuantía máxima de esta prestación será la siguiente, hasta que se regule reglamentariamente: $442,59 € /$ mes (grado III, nivel 2), 354,43€/mes (grado III, nivel 1), 286,66€/mes (grado II, nivel 2), $255,77 € /$ mes (grado II, nivel 1) o $152 € /$ mes (grado I, nivel 2). A aquellos solicitantes que no tengan reconocido grado y nivel de dependencia, así como a los nuevos solicitantes, les será aplicada una cuantía más reducida $-387,64 € /$ mes (grado III), 268,79€/mes (grado II) $0153 € /$ mes (grado I)- hasta que se regule reglamentariamente (disposición transitoria décima).

${ }^{6}$ Para el ejercicio 2008, el Real Decreto 179/2008, de 8 de febrero, por el que se modifica el Real Decreto 6/2008, actualizó las cuantías señaladas para el nivel mínimo de protección garantizado por la Administración General del Estado para cada beneficiario del sistema en situación de grado III (gran dependencia), de conformidad con el incremento del 3,50 \% del IPREM, hasta 258,75 (grado III, nivel 2) y 175,95 euros (grado III, nivel 1). Del mismo modo, estableció la cuantía para el nivel mínimo de protección garantizado para los beneficiarios valorados con el grado II, nivel 2 (dependencia severa) en 100 euros mensuales. Para el ejercicio 2009, el Real Decreto 74/2009, actualizó las cuantías de conformidad con el incremento del $2 \%$ del 
Con la aprobación del Real Decreto Ley 20/2011, de 30 de diciembre, de Medidas Urgentes en Materia Presupuestaria, Tributaria y Financiera para la Corrección del Déficit Público, y a posteriori, se incumple la actualización anual del nivel mínimo de protección y el calendario de aplicación progresiva regulado en la disposición final primera de la Ley de Dependencia, que se pospone hasta mediados de $2015^{7}$. Así, en el ejercicio 2012 las cuantías de la asignación a las comunidades autónomas del nivel mínimo de protección previsto en el artículo 9 de la Ley 39/2006, lejos de ser actualizadas, se redujeron considerablemente y no a todos los dependientes por igual, sino en función de la fecha en la que hubiera tenido lugar la resolución del grado y nivel de dependencia: la disposición transitoria undécima del Real Decreto Ley 20/2012, de 13 de julio, establece que los beneficiarios que tengan resolución de grado y nivel de dependencia reconocido a la entrada en vigor de este real decreto ley recibirán las cuantías correspondientes al nivel mínimo de protección garantizado por el Estado (Tabla 1). En cambio, para los beneficiarios que no tengan resolución de reconocimiento de la situación de dependencia a la entrada en vigor de este real decreto ley, la aportación del Estado es aún menor, concretada en cantidades -ahora sólo por gradosque resultan ser un promedio entre el nivel máximo y el mínimo (Tabla 1).

Dichas cuantías no han sido modificadas por el Real Decreto 1050/2013, de 27 de diciembre, regulador ción de la dependencia del nivel mínimo de protección, ya que en su artículo 4 establece que la aportación del Estado para la financiación de este nivel será, para cada grado, la establecida en la disposición transitoria undécima del Real Decreto Ley 20/2012, de 13 de julio.

Como se ha podido comprobar, las aportaciones del nivel mínimo de protección para cada grado se han reducido, tras el Real Decreto Ley, un $13 \%$ de media ${ }^{8}$.

\subsubsection{Suspensión del nivel intermedio de financiación del sistema de dependencia}

El segundo nivel de protección del sistema de dependencia es el compartido entre la Administración General del Estado y la de cada una de las comunidades autónomas (art. 10.1 de la Ley de Dependencia) dentro del marco de cooperación interadministrativa, acordado en el seno del Consejo Territorial de Servicios Sociales y del SAAD, y desarrollado mediante convenios de colaboración?.

En los convenios se han venido determinando las obligaciones asumidas por cada una de las partes (Administración General del Estado y Administración Autonómica) para financiar los servicios y prestaciones del sistema, teniendo en cuenta las características concretas de cada comunidad autónoma en cuanto a población potencialmente dependiente (los primeros años) o la población dependiente

Tabla 1. Cuantías mínimas de la prestación por dependencia fijadas por el Real Decreto Ley 20/2012, según fecha de la resolu-

\begin{tabular}{|c|c|c|c|}
\hline \multicolumn{2}{|c|}{ Anterior al 15-VII-2012 } & \multicolumn{2}{|c|}{ Posterior al 14-VII-2012 } \\
\hline Nivel de dependencia & Cuantía (€) & Nivel de dependencia & Cuantía (€) \\
\hline Grado III. Gran dependencia, nivel 2 & 231,28 & \multirow{2}{*}{ Grado III. Gran dependencia } & \multirow{2}{*}{177,86} \\
\hline Grado III. Gran dependencia, nivel 1 & 157,26 & & \\
\hline Grado II. Dependencia severa, nivel 2 & 89,38 & \multirow{2}{*}{ Grado II. Dependencia severa } & \multirow{2}{*}{82,84} \\
\hline Grado II. Dependencia severa, nivel 1 & 61,36 & & \\
\hline Grado I. Dependencia moderada, nivel 2 & 52,06 & Grado I. Dependencia moderada & 44,33 \\
\hline
\end{tabular}

Fuente: Elaboración propia a partir del Real Decreto Ley 20/2012.

IPREM, hasta 263,93 (grado III, nivel 2), 179,97 (grado III, nivel I) y 102,00 euros mensuales (grado II, nivel 2). Igualmente, estableció en 70 euros mensuales la cuantía correspondiente a los dependientes valorados en el grado II, nivel 1. Para el ejercicio 2010, último año de actualización, el Real Decreto 373/2010, de 26 de marzo, con arreglo al incremento del $1 \%$ del indicador público de renta de efectos múltiples, actualiza las cuantías, que quedan en 166,57 (grado III, nivel 2), 181,26 (grado III, nivel 1), 103,20 (grado II, nivel 2) y 70,70 euros (grado II, nivel 1).

7 Como se observa, la actualización de las cuantías del nivel mínimo de protección a cargo exclusivo del Estado tan sólo se cumplió durante los tres primeros años de vigencia de la Ley de Dependencia. No obstante, ya se preveía un estancamiento, porque durante este periodo el incremento de las cuantías fue progresivamente menor: del $3,5 \%$ para el ejercicio 2008 , del $2 \%$ para el 2009 , del $1 \%$ para el 2010 y del o \% para los ejercicios siguientes.
${ }^{8}$ En términos anuales, la financiación del nivel mínimo ha pasado de 1.634 millones de euros a 1.418 millones de euros, lo que supone una reducción de $\mathbf{2 1 6}$ millones de euros al año. Véase el análisis de los cambios acaecidos en el nivel mínimo de protección garantizado por la Administración General del Estado en Vilaplana (2012: 40).

${ }_{9}$ A tal efecto, la disposición adicional cuarta de la Ley de Dependencia establece que "en el plazo máximo de tres meses desde su constitución, el Consejo Territorial acordará el marco de cooperación interadministrativa para el desarrollo de la Ley que el artículo 10 prevé". A este respecto, el Consejo Económico y Social advertía de que "es fundamental una adecuada articulación del régimen de cooperación y financiación entre la Administración General del Estado y las Comunidades Autónomas, a fin de que, mediante los convenios que se suscriban al efecto, se dote de eficiencia y efectividad al desarrollo y aplicación de las prestaciones y servicios" (2006: 9). 
ya evaluada (los años posteriores) ${ }^{10}$, la dispersión geográfica, la insularidad, los emigrantes retornados $u$ otros factores, con el objeto de respetar el principio de equidad en la distribución territorial de los recursos económicos (arts. 10.4 y 32.3 de la Ley de Dependencia) ${ }^{11}$. Para ello, debe tenerse en cuenta que la aportación de la comunidad autónoma en cuestión será, cada año, al menos igual a la de la Administración General del Estado.

En efecto, la financiación ha venido siendo asumida, respetando un cierto equilibrio ${ }^{12}$, por ambas Administraciones, tal y como exige la Ley de Dependencia, hasta el ejercicio $2010^{13}$. Con la aprobación de la Ley de Presupuestos Generales para 2012 (Ley 2/2012, de 29 de junio), sin embargo, se suspende durante todo ese año la posibilidad de realizar convenios entre el Estado y las comunidades autónomas para determinar la financiación del nivel intermedio. Dicho periodo suspensivo se amplía tres años más con las leyes de Presupuestos Generales del Estado para 2013 (Ley 17/2012, de 27 de diciembre), para 2014 (Ley 22/2013, de 23 de diciembre) y para 2015 (Ley $36 / 2014$, de 26 de diciembre), de modo que desaparece la partida presupuestaria correspondiente a la financiación del nivel acordado por parte de la Administración General del Estado.

${ }^{10}$ Durante los primeros años de aplicación de la Ley, la variable relativa a la población dependiente no se obtuvo exclusivamente a partir del número de dependientes reconocidos, sino que también se otorgaba un peso importante (que progresivamente ha ido disminuyendo) a la población potencialmente dependiente (estimada). Esta circunstancia ha provocado que el sistema de financiación no se ajuste a las peculiaridades reales de la población dependiente, de forma que algunas comunidades autónomas han tenido déficit de protección, mientras que otras han sido excedentarias (Correa Gómez, Montero Granados y Jiménez Aguilera, 2011: 3).

${ }^{11} \mathrm{~A}$ este respecto, el Consejo Económico y Social advirtió de que "en el momento de planificar los recursos, así como de concretar el contenido del derecho a las condiciones de acceso al mismo, debería tomarse en consideración la circunstancia de que buena parte de las personas con problemas de autonomía personal o dependencia (en general, personas con edad avanzada) residen en municipios rurales, cuyas peculiaridades socioeconómicas difieren notablemente de los núcleos urbanos y cuya dispersión geográfica genera graves dificultades de accesibilidad a los recursos sociales, por lo que junto con el volumen de población dependiente es necesario considerar su distribución en los distintos tipos de municipios y la dispersión de estos últimos, a fin de lograr el reparto equitativo de los recursos y la igualdad real en el acceso a los mismos" (2006: 9). Dicha advertencia ha sido tenida en cuenta en los diferentes acuerdos, pero hasta la fecha no se han conseguido los resultados esperados.

${ }_{12}$ Los datos reflejan que la contribución de la Administración General del Estado (AGE) y de las comunidades autónomas al sistema de dependencia ha venido siendo equilibrada. No obstante, de un análisis comparativo de las aportaciones realizadas por las distintas administraciones se extraen algunas desviaciones. Es el caso, por ejemplo, del País Vasco, La Rioja y Andalucía, donde el aporte autonómico es superior al realizado en su territorio por la AGE; en el otro extremo se sitúan Madrid, la Comunidad Valenciana o Canarias, que realizaran una aportación al sistema inferior a la efectuada por la AGE, una vez más, reflejo de los criterios políticos de Gobierno (Ministerio de Sanidad, Política Social e Igualdad, 2011: 198).

${ }^{13}$ Según los datos extraídos del Informe del Gobierno para la Evaluación de la Ley de Promoción de la Autonomía Personal y Atención a las Personas en Situación de Dependencia (Ministerio de Sanidad, Política Social e Igualdad, 2011: 197), donde no se incluyen las cifras relativas a las cuotas de Seguridad Social de las personas cuidadoras no profesionales, cuyo pago es asumido por la Administración General del Estado. En caso de incluirse, la aportación financiera de ésta aumentaría proporcionalmente.
Por lo tanto, el Estado sólo está contribuyendo a la financiación del sistema de dependencia con la cuantía correspondiente al nivel mínimo garantizado por el Estado, que, como se ha visto, ha sido notablemente reducida, por lo que recae sobre las comunidades autónomas el esfuerzo y responsabilidad de aumentar su aportación al sistema si desean mantener el mismo nivel prestacional, asumiendo en exclusiva el coste de prestaciones, que, con anterioridad, era compartido con el Estado (González Ortega, 2013: 11 y 12; Colás Neila, 2013: 104).

\subsubsection{Incremento de la participación de los beneficiarios en el coste del sistema}

El artículo 33 de la Ley de Dependencia regula la participación de las personas beneficiarias en el coste de las prestaciones, uno de sus aspectos más criticados. Sin embargo, la precisión de los criterios generales establecidos se remite al Consejo Territorial, que, de conformidad con lo establecido en el artículo 8.2d, alcanzó el Acuerdo de 27 de noviembre de 2008 sobre determinación de la capacidad económica de las personas beneficiarias (Resolución de 2 de diciembre de 2008), así como los criterios de participación de éstos en los costes del SAAD, modificado posteriormente por el Acuerdo de 10 de julio de 2012 (Resolución de 13 de julio de 2012). A través de este segundo acuerdo, se introducen modificaciones sustanciales en el modelo de copago acordado previamente, con la presumible finalidad de garantizar el principio de igualdad en esta materia y de mejorar el Sistema para la Autonomía y Atención a la Dependencia en su conjunto, al mismo tiempo que se aumenta la participación económica de los usuarios (Durán Bernardino, 2014).

El análisis del modelo de copago de la dependencia establecido en la Ley de Dependencia y desarrollado mediante el Acuerdo de 10 de julio de 2012 (Resolución de 13 de julio de 2012) muestra que es un modelo con dos objetivos claros, uno explícito y otro tácito: el primero es conferir uniformidad en las cuotas con independencia de la ubicación del domicilio de la persona beneficiaria; en cambio, ya se puede confirmar la operatividad de su segundo objetivo, el recaudatorio. Es una realidad compartida por todo el territorio nacional que, en materia de dependencia, la aportación de los beneficiarios al sistema, lejos de disminuir, ha ido aumentando año tras año a medida que se reducía la aportación de las administraciones públicas, como se comprueba en el Gráfico $1^{14}$.

${ }^{14}$ En 2009, la aportación de los usuarios representó un $10 \%$ de la financiación total del sistema de dependencia (es decir, una aportación de 387,4 millones de euros); en 2010, un $12 \%(676,08$ millones de euros); en 2011, un $14 \%$ (912,38 millones de euros); y en 2012 representó un $16 \%$ de la financiación total (1.051,2 millones de euros) [Barriga, L. A. et al., 2013]. 


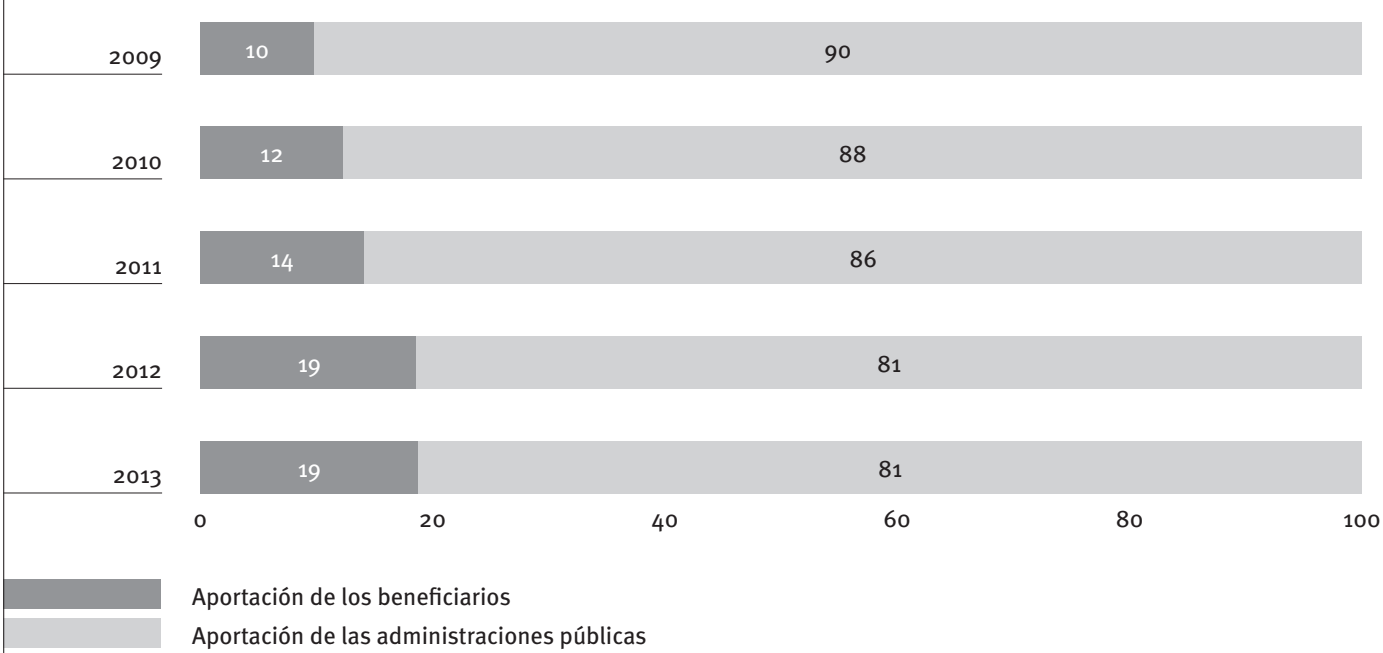

Fuente: Elaboración propia a partir Barriga et al. (2014).

La aplicación de los criterios para determinar el copago establecidos en la Resolución de 13 de julio de 2012 no sólo continúa en esta dirección, sino que implica un importante incremento de su cuantía, de forma que la recaudación media pasa a ser del $50 \%$ del coste de las prestaciones, aproximadamente (Montserrat Codorniu y Montejo Sarrias, 2011: 86).

No obstante, cabe advertir que la participación económica del beneficiario en el coste de las prestaciones todavía no ha sido implantada por el Estado mediante disposición general; por lo tanto, es nula toda la normativa autonómica aprobada en desarrollo del Acuerdo del Consejo Territorial de 2012 en relación a los criterios de participación del beneficiario en el coste del sistema. Así lo ha determinado la Audiencia Nacional (sentencia del 25 de febrero de 2011, RJCA 2011/157), el Tribunal Supremo (sentencia del 3 de noviembre de 2011) y la Sala de lo Contencioso Administrativo, Sección 4 ${ }^{\mathrm{a}}$, del Tribunal Superior de Justicia (sentencia del 13 de marzo de 2014, AS 107/14).

\section{Paralización del calendario de aplicación progresiva de la Ley}

Con la finalidad de dar prioridad a las personas con mayor grado de dependencia en el acceso a los recursos existentes, y al mismo tiempo, corregir el déficit público, dada la situación económica y presupuestaria, se modificó el calendario de aplicación de la Ley 39/2006, de 14 de diciembre (disposición final primera) en dos ocasiones.

El Real Decreto Ley 20/2011, de 30 de diciembre, de Medidas Urgentes en Materia Presupuestaria, Tributaria y Financiera para la Corrección del Défi- cit Público, introduce una nueva redacción en la disposición final primera de la Ley de Dependencia, que afecta directamente a los dependientes moderados, en sus dos niveles. Con respecto al nivel 2 de dependencia, marca una clara diferenciación entre quienes hayan sido valorados con grado I, nivel 2 antes del 31 de diciembre de 2011 y quienes en dicha fecha, pese a haber presentado la solicitud, no hayan sido valorados. En el primer caso, los sujetos se consideran incluidos en el campo de aplicación de la Ley y con derecho a la prestación una vez resuelto su reconocimiento; en cambio, en el segundo caso, los sujetos quedan excluidos temporalmente del sistema, ya que aquellas personas valoradas en grado I, nivel 2 a partir del 31 de diciembre de 2011 deberían esperar hasta el 1 de enero de 2013 para tener derecho a la prestación. Esta congelación del derecho y el bloqueo del procedimiento supone un retraso de dos años en la aplicación de la Ley para los dependientes moderados del máximo nivel, lo cual se traduce en un ahorro generalizado para el sistema, de desigual repercusión en las comunidades autónomas (González Ortega, 2013: 10).

En lo que se refiere a los moderados del nivel 1, el Real Decreto altera una vez más lo dispuesto en la disposición final primera, al retrasar un año la aplicación de la Ley a aquellos dependientes valorados en el grado I, nivel 1, que no se incorporarán al sistema hasta el 1 de enero de $2014^{15}$. Tan sólo seis meses más tarde de la modificación expuesta, el aplazamiento establecido para el nivel 1 se hace extensible al nivel 2 por la Ley $2 / 2012$, de 29 de junio, de Presupuestos Generales del Estado, en aquellos casos en

${ }^{15}$ Concretamente, 29.838 personas con dependencia moderada reconocida no recibieron la ayuda a la que tenían derecho por la introducción de esta medida. Tan sólo la recibieron aquellas que tenían reconocida la concreta prestación. 
los que no se les hubiera reconocido la prestación a 15 de julio de 2012.

Es aquí donde incide el Real Decreto Ley 20/2012, de 13 de julio, de Medidas para Garantizar la Estabilidad Presupuestaria y de Fomento de la Competitividad, al establecer que todos los dependientes moderados a los que no se les haya reconocido la prestación sólo podrán comenzar a disfrutarla a partir del 1 de julio de 2015. Así, el derecho efectivo a las prestaciones de dependencia de quienes fueron valorados en el grado I de dependencia moderada, nivel 2 , sin tener reconocida la concreta prestación se producirá a partir de esa fecha, al igual que la de aquellos que hayan sido valorados en el grado I, nivel $1^{16}$. Por lo tanto, el apartado 1 de la disposición final primera de la Ley de Dependencia queda redactado, respecto a la implantación que restaba, en los siguientes términos (Molina González-Pumariega, 2012: 115):

- En el quinto año de implantación de la Ley, que finaliza el 31 de diciembre de 2011, a quienes sean valorados en el grado I de dependencia moderada, nivel 2 , y se les haya reconocido la concreta prestación.

- A partir del 1 de julio de 2015, al resto de quienes fueron valorados en el grado I, de dependencia moderada, nivel 2.

- A partir del 1 de julio de 2015 a quienes hayan sido valorados en el grado I, nivel 1, o sean valorados en el grado I, de dependencia moderada.

Por lo tanto, la Ley de Dependencia queda en suspenso durante tres años y medio para este colectivo, que ve cómo su derecho subjetivo de ciudadanía no se hace efectivo (Arenas Viruez, 2013).

\section{Valoración crítica: consecuencias de las medidas adoptadas}

Las medidas analizadas han tenido una grave incidencia en la protección de las personas en situación de dependencia y en sus familiares. Bajo la perspectiva de los tres años transcurridos, es el momento de extraer los efectos y consecuencias de su implantación, y de comprobar si realmente se ha conseguido el objetivo preconizado por las disposiciones normativas -sostenibilidad, homogeneidad, viabilidad-o si, por el contrario, han derivado en una notable disminución del nivel de cobertura del sistema de dependencia.

${ }^{16}$ A 1 de diciembre de 2012, se habían emitido un total de 1.538.561 dictámenes, 400.731 corresponden al grado III de dependencia, 460.195 al grado II y 140.326 al grado I. Sin embargo, el número de beneficiarios con derecho a prestación era de 1.001.151. Esto significa que, en la fecha indicada había 537.410 personas con grado de dependencia reconocido que no eran beneficiarias con derecho a prestación, y lo que es más preocupante, que su incorporación al sistema no se producirá, en principio, hasta mediados de 2015.

\subsection{Retroceso en la acción protectora del sistema}

Formalmente, a la prestación económica para cuidados en el entorno familiar (PECEF) se le otorga un carácter excepcional, situándola en una posición claramente marginal respecto al resto de prestaciones. Sin embargo, durante los ocho primeros años de aplicación del Sistema para la Autonomía y Atención a la Dependencia, ha sido la protagonista indiscutible del sistema, lo que ha provocado la adopción de medidas mediante las que se ha perseguido un cambio de dirección en el modelo imperante hasta ahora, a favor del modelo originario, basado en prestaciones de servicios y cuidados profesionales. Y ello a pesar de que, para garantizar la viabilidad de este modelo basado en servicios, se requiere una elevada inversión económica, ante la deficitaria red pública de servicios sociales con la que cuenta actualmente España. Una inversión que, por otro lado, no se contempló en los Presupuestos Generales del Estado de 2013 y 2014, ni se ha contemplado en los de $\mathbf{2 0 1 5}$, en los que se continúa suprimiendo el nivel acordado y disminuye el nivel mínimo de financiación.

Tres años después de la introducción de estas medidas descentivadoras de las prestaciones económicas para cuidados en el entorno familiar, se puede comprobar, a través de los datos estadísticos proporcionados mensualmente por el Instituto de Mayores y Servicios Sociales (Imserso), el impacto real que han tenido las medidas emprendidas en julio de 2012 en las prestaciones reconocidas a los beneficiarios del sistema. En el Gráfico 2, se representa la fluctuación del número total de prestaciones reconocidas desde 2008 hasta 2015 , así como la distribución y representatividad de las distintas prestaciones que conforman el sistema de dependencia, cuyo análisis permite extraer los siguientes datos y conclusiones:

- En junio de 2012, mes anterior a la aprobación de las reformas estudiadas, el número de prestaciones económicas para cuidados en el entorno familiar era de 427.747. Tres años después, en mayo de 2015, el reconocimiento de esta prestación se ha reducido a 363.217, es decir, el 38,47\% del total de prestaciones reconocidas a los beneficiarios del sistema, frente al 45,16\% anterior a la reforma.

- Esta reducción en la prestación económica para cuidado familiar debería implicar un incremento en las prestaciones de servicios reconocidas, para cumplir así con el objetivo principal establecido en el Real Decreto 20/2012; sin embargo, no ha sido así. Si bien es cierto que alguna de las prestaciones de servicios han experimentado un leve incremento en los dos últimos años (servicio de prevención de las situaciones de dependencia y de promoción de la autonomía personal, centro de día y de noche, atención residencial), no se puede afirmar, en virtud a los datos globales analizados, que la reducción de la 'prestación estrella' hasta el momento haya implicado un incremento del resto. Afirmarlo sería una falacia insostenible, ya que la consecución de este 
resultado ha ido acompañada de una importante reducción del número total de prestaciones reconocidas.

- Si atendemos al total de prestaciones, comprobamos que se ha reducido en 2.879 a raíz de la reforma, pasando de 947.086 en junio de 2012 a 944.207 en mayo de 2015 .

- También se puede extraer lo que ya se preveía (Durán Bernardino, 2014): las medidas adoptadas derivan en una solución intermedia, la prestación económica vinculada al servicio, que ha sufrido un incremento de 13.849 prestaciones en los últimos tres años, pues resulta más rentable el reconocimiento de una prestación económica al beneficiario, dirigida a adquirir el servicio en un centro privatizado. Sin duda, esta solución marcará las diferencias en el acceso a las prestaciones (ibídem: 15-43).

Los datos analizados permiten concluir que, al restringir la prestación económica para cuidados en el entorno familiar tan sólo se ha conseguido uno de los objetivos perseguidos, la reducción del gasto público, pues ha disminuido el número de prestaciones reconocidas a los beneficiarios, a pesar de que existen miles de personas a la espera de percibir la prestación que les ha sido reconocida y de que miles de dependientes fallecen sin llegar a percibir la prestación a la que tienen derecho. La consecuencia principal de las medidas adoptadas es que, lejos de suponer un aumento de las prestaciones de servicios concedidas, implican un recorte más de los que viene mermando la implantación de la Ley de Dependencia (Molero Marañón, 2012: 157-159), que deriva en una alarmante desprotección a la que se enfrentan día tras día las personas en situación de dependencia y sus familias.

\subsection{Financiación insuficiente}

Como se ha analizado, en los últimos tres años la aportación de la Administración General del Estado se ha reducido -tras la suspensión del nivel intermedio de financiación y la notoria disminución del nivel mínimo garantizado por el Estado-, con la presumible intención de generar un ahorro en el gasto de la Administración General del Estado para alcanzar un reequilibrio sostenible del sistema, sin tener en cuenta lo que ya se advertía en el informe de Gobierno para la evaluación de la Ley de Dependencia (Ministerio de Sanidad, Política Social e Igualdad, 2011), donde se ponía de manifiesto la necesidad de que las administraciones públicas incrementaran sus aportaciones para conseguir la sostenibilidad económica y financiera del Sistema para la Autonomía y Atención a la Dependencia ${ }^{17}$.

Gráfico 2. Prestaciones reconocidas por el Sistema para la Autonomía y Atención a la Dependencia, por tipo de prestación (2008-2015)

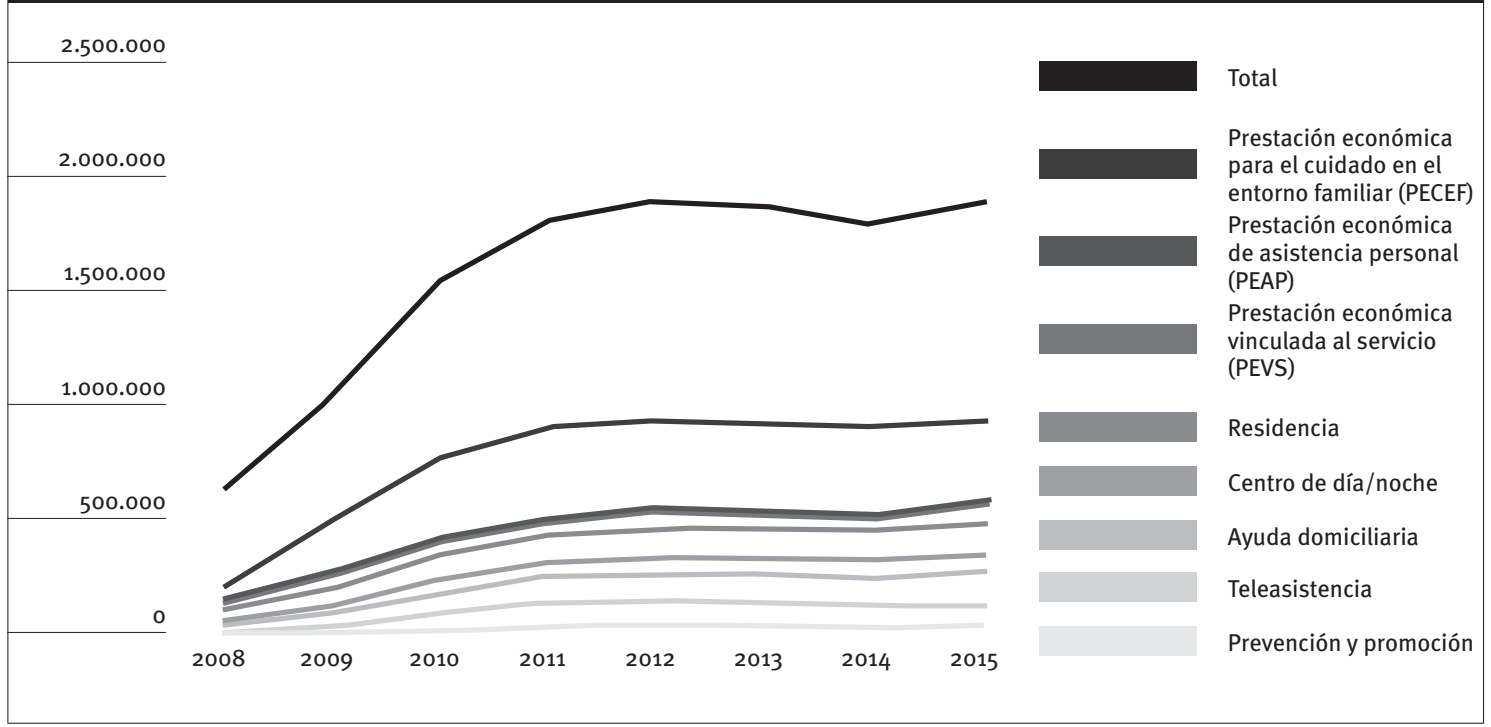

Fuente: Elaboración propia a partir de las estadísticas del Sistema para la Autonomía y Atención a la Dependencia (Imserso).

${ }_{17}$ A este respecto, el informe publicado en mayo de 2012 por el Comité de Derechos Económicos, Sociales y Culturales de la ONU considera que tales derechos no son rescindibles en épocas de crisis económica. Con anterioridad, el Consejo de Derechos Humanos fue más allá, al advertir a los Estados de que las crisis económicas y financieras mundiales no disminuyen la responsabilidad de las autoridades nacionales y de la comunidad internacional en la realización de los derechos humanos, ya que los principios de derechos humanos son los que deben guiar cualquier estrategia de recuperación para hacer frente a una recesión económica. 
Tras lo acontecido, no se puede negar que las reformas han dados sus frutos: se ha ahorrado en el gasto público. Ahora bien, igualmente cierto es que ese ahorro se ha conseguido a costa de la desprotección de las personas en situación de dependencia, no garantizándose el derecho que en su día les fue reconocido. Así se comprueba en los datos que integran la Tabla 2, en la que se realiza un análisis comparativo de los datos de gestión del sistema de dependencia y en los que debe prestarse especial atención a la notoria reducción experimentada en los tres años transcurridos desde la aprobación del Real Decreto de 2012, en general, en el número de personas dependientes que se incorporan al sistema y, en particular, en el número de beneficiarios con derecho efectivo a prestación y en el número de beneficiarios con prestación concedida.

En junio de 2012, el número total de personas dependientes reconocidas con expediente activo era de 1.276.442. Como resultado de las medidas de recorte inversor en la política de promoción de la autonomía personal y atención a la dependencia, a partir de agosto de 2012 el sistema empezó a arrojar saldos negativos, siendo actualmente (según el último dato publicado, de mayo 2015) de 1.189.375 personas en situación de dependencia.

Ahora bien, como se sabe, una cosa es tener el reconocimiento de dependencia dictaminada en alguno de sus grados y otra muy distinta ser beneficiario con derecho efectivo a prestación, pues el derecho a ser atendido depende del calendario de aplicación progresiva de la Ley de Dependencia. Por ello, entre las personas en situación de dependencia hay que diferenciar aquellos beneficiarios con derecho efectivo a prestación de aquellos que no lo tienen (312.025, a 31 de mayo 2015). Si comparamos el último dato de personas dependientes con derecho a atención (877.350, a 31 de mayo 2015) con el dato de mediados de 2012 (junio, 1.028.455) o con el de finales de 2011 (diciembre, 1.053.957), se comprueba que las cifras de beneficiarios con derecho efectivo a prestación no han dejado de disminuir. Ello se debe a las bajas de los titulares por fallecimiento y a la leve incorporación de nuevos dependientes al sistema, que no compensa las bajas producidas.

Llegados a este punto, el dato que más puede interesar es el número de personas atendidas y desatendidas por el sistema. A mediados de 2012, el número de beneficiarios con alguna prestación económica o de servicio concedida ascendía a 758.364. Los últimos datos disponibles, a 31 de mayo de $\mathbf{2 0 1 5}$, ofrecen una cifra de atendidos de 745.159 , es decir, 13.205 personas menos.

Haciendo balance: se reduce el número de personas dependientes incorporadas al sistema (en 87.067 personas en los últimos tres años), el número de beneficiarios con derecho efectivo a prestación disminuye (en 151.105 personas en el mismo margen de tiempo) e igualmente arroja resultados negativos el número de beneficiarios con prestación concedida (13.205 menos que antes de la reforma $)^{18}$. Estos datos no sólo permiten confirmar el estancamiento total del sistema, sino que también llevan a hablar de retroceso en la acción protectora como resultado de la importante reducción en el número de personas atendidas.

El síntoma más claro del colapso del sistema es el número de personas desatendidas, que según el último dato del que se dispone (mayo 2015), asciende a 132.191 personas. Si lo comparamos con el número de personas desatendidas hace tres años (270.091 en junio de 2012), podría pensarse que se ha conseguido la tan deseada y proclamada reducción de la lista de espera; sin embargo, dicha reducción no se debe al incremento de personas atendidas, como ya se ha comprobado y se muestra en el Gráfico 3, sino a las reformas introducidas en los últimos años: la retirada del derecho a atención a las personas con grado I (nivel 2) que no estuvieran recibiendo atención a 31 de diciembre de 2011 y el fallecimiento de las personas que esperaban a ser atendidas.

Tabla 2. Resumen comparativo de los datos globales de gestión del Sistema para la Autonomía y la Atención a la Dependencia (2012-2015)

\begin{tabular}{|c|c|c|c|c|}
\hline & \multicolumn{2}{|c|}{ A junio de 2012} & \multicolumn{2}{|c|}{ A mayo de 2015} \\
\hline Total solicitudes & \multicolumn{2}{|c|}{1.630 .720} & \multicolumn{2}{|c|}{1.571 .742} \\
\hline & \multicolumn{2}{|c|}{1.520 .263} & \multicolumn{2}{|c|}{1.498 .582} \\
\hline Personas que han sido valoradas & $\begin{array}{c}1.276 .442 \\
\text { (en situación de } \\
\text { dependencia) }\end{array}$ & $\begin{array}{c}243.822 \\
\text { (no dependientes) }\end{array}$ & $\begin{array}{c}1.189 .375 \\
\text { (en situación de } \\
\text { dependientes) }\end{array}$ & $\begin{array}{c}306.114 \\
\text { (no dependientes) }\end{array}$ \\
\hline $\begin{array}{l}\text { Personas pendientes del derecho } \\
\text { efectivo a prestación }\end{array}$ & \multicolumn{2}{|c|}{247.987} & \multicolumn{2}{|c|}{312.025} \\
\hline \multirow{2}{*}{$\begin{array}{l}\text { Beneficiarios con derecho efectivo } \\
\text { a prestación }\end{array}$} & \multicolumn{2}{|c|}{1.028 .455} & \multicolumn{2}{|c|}{$877 \cdot 350$} \\
\hline & $\begin{array}{c}758.364 \\
\text { (prestación concedida) }\end{array}$ & $\begin{array}{c}270.091 \\
\text { (prestación en trámite) }\end{array}$ & $\begin{array}{c}745.159 \\
\text { (prestación concedida) }\end{array}$ & $\begin{array}{c}132.191 \\
\text { (prestación en trámite) }\end{array}$ \\
\hline
\end{tabular}

Fuente: Elaboración propia a través de las estadísticas del Sistema para la Autonomía y Atención a la Dependencia (Imserso).

${ }^{18}$ Véase un análisis más exhaustivo en el XIII Dictamen del Observatorio de la Dependencia (Barriga et al., 2014). 


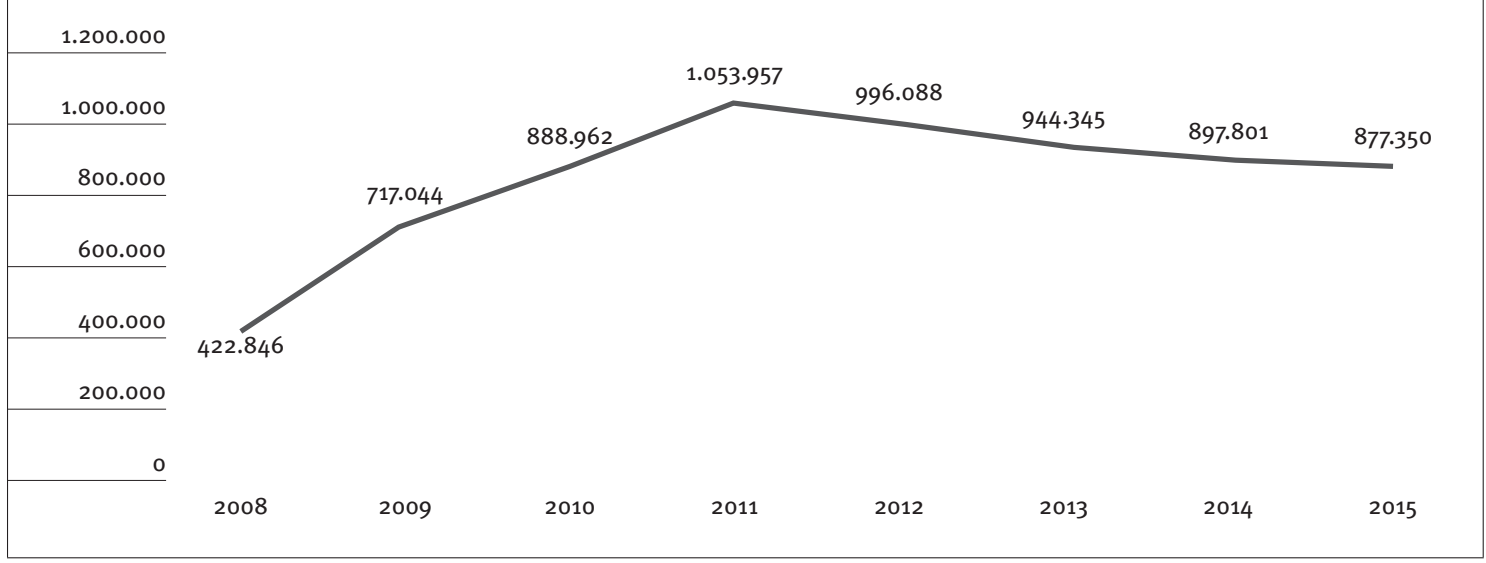

Fuente: Elaboración propia a partir de las estadísticas del Sistema para la Autonomía y Atención a la Dependencia (Imserso).

Los resultados expuestos ponen de manifiesto cómo las respuestas que se le están dando a la profunda recesión económica, desde políticas neoliberales que destruyen a su paso nuestro consolidado Estado de bienestar, están provocando la paralización de un sistema aún por consolidar y la derogación implícita de una ley muy esperada. Actualmente, la sostenibilidad del sistema es uno de los aspectos que más preocupa, pues la financiación es claramente insuficiente y la crisis financiera y económica no permite vislumbrar un cambio de dirección a corto o medio plazo.

\subsection{Reducción del número de personas con derecho a ser atendidas}

El calendario de aplicación de la Ley de Dependencia se ha paralizado y aplazado de forma progresiva en los últimos años - primero con la Ley 20/2011, después con la Ley 2/2012 y en tercer lugar con el Real Decreto 20/2012-, con la finalidad de dar prioridad a las personas con mayor grado de dependencia para acceder a los recursos disponibles. Con la perspectiva de los tres años transcurridos, es el momento de comprobar si efectivamente la finalidad

Gráfico 4. Evolución de los grados de dependencia dictaminados (2008-2015)

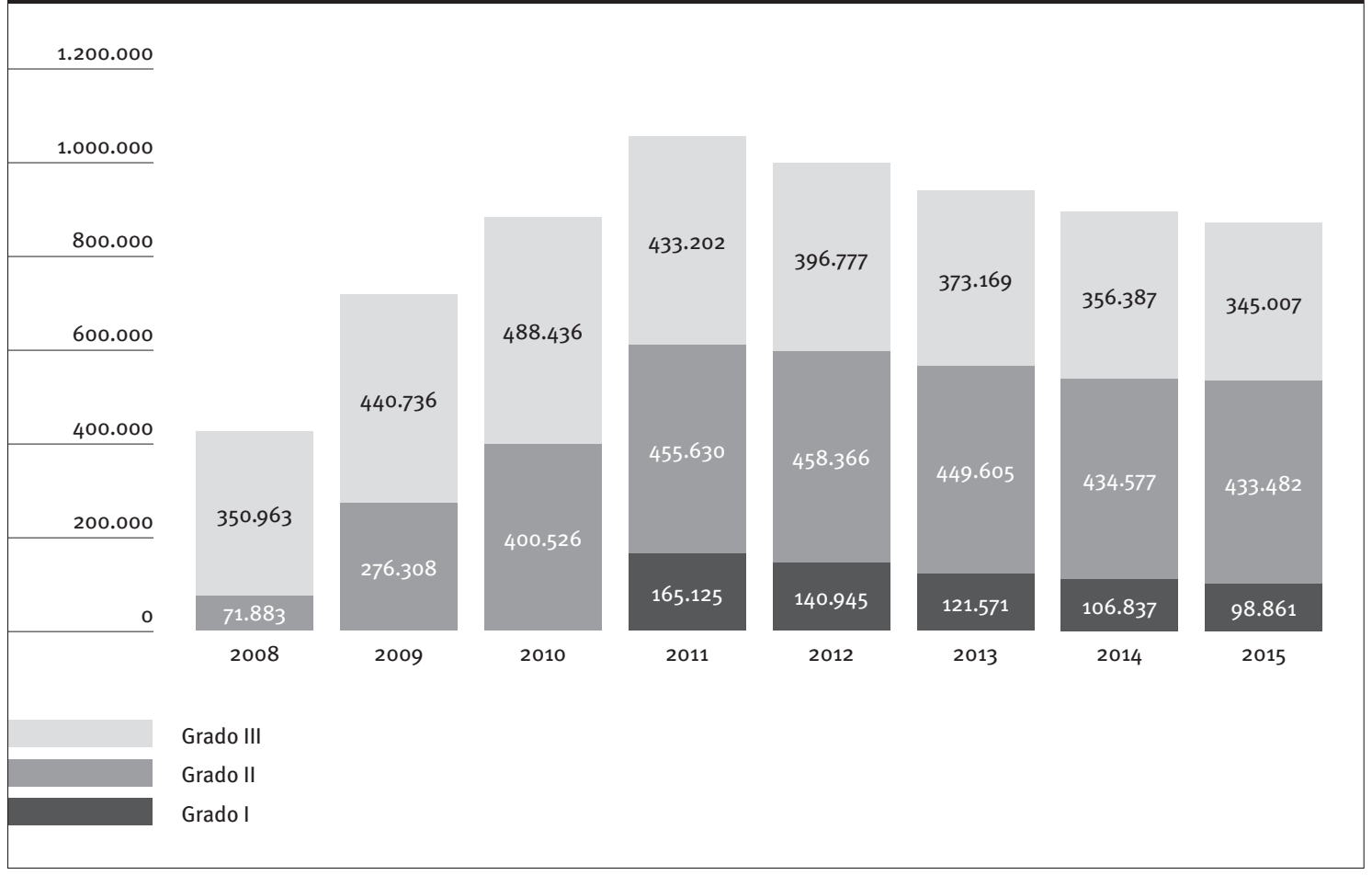

Fuente: Elaboración propia a partir de las estadísticas del Sistema para la Autonomía y Atención a la Dependencia (Imserso). 
principal de las medidas adoptadas era dar prioridad a aquellas personas con una situación de dependencia más grave ${ }^{19}$, y no la de reducir costes excluyendo del sistema a personas que no dejan de ser calificadas como dependientes ${ }^{20}$.

El punto de inflexión que se aprecia en el Gráfico 3 coincide con la entrada en vigor del Real Decreto Ley 20/2011, de 30 de diciembre, por el que se suspendió la incorporación al sistema de las personas dependientes con grado I, nivel 2. Por otro lado, si se observa la evolución de titulares de derecho por grados de dependencia (Gráfico 4), se comprueba cómo la disminución de personas con derecho a atención no sólo afecta al grado I, dependencia moderada, sino que se extiende a todos los grados de dependencia desde las reformas adoptadas. En diciembre de 2011, el número de personas en situación de dependencia era de 1.053.957 personas, de las cuales 433.202 fueron dictaminadas en grado III, 455.630 en grado II y 165.125 en grado I. En cambio, tres años después, el número de personas en situación de dependencia ha disminuido a 877.350: 345.007 dictaminadas en grado III, 433.482 en grado II y 98.861 en grado I.

Por lo tanto, el sacrificio de los dependientes moderados al aplazarse su incorporación al sistema no ha contribuido a que los grandes dependientes y dependientes severos accedan al sistema. Lejos de ser así, y una vez más primando la finalidad 'real' de las reformas (corregir el déficit público), el número de personas con derecho a atención no ha dejado de decaer en los últimos años, lo que ha afectado a los tres grados de dependencia.

Tras tres años excluidos de cualquier tipo de prestación, para el 1 de julio de 2015 está previsto que los dependientes moderados se incorporen al sistema. Sin embargo, es llamativo que dicha incorporación no se prevea en los Presupuestos Generales del Estado aprobados para 2015. En ellos, el Gobierno consigna una partida de gasto mínimo idéntica a la de 2014, de 1.087.179.000 euros, cantidad difícilmente suficiente para atender a los actuales 745.159 beneficiarios con prestación concedida, incorporar a los 132.191 que se encuentran en lista de espera y, además, responder a los costes que supone la incorporación de los dependientes moderados que están fuera del sistema, lo que hace prever un nuevo aplazamiento. El pasado 28 de noviembre, el Consejo de Ministros autorizó una nueva aplicación del Fondo de Contingencia, al destinar 57.708.102,76 euros para financiar un suplemento de crédito para atender el nivel mínimo de protección garantizado por la AGE. Pese a dicho incremento, desde algunos ámbitos ya se advierte que dicho fondo es insuficiente.

19 En cualquier caso, esta reforma no afecta a los grandes dependientes ni a los dependientes severos, que podrán continuar solicitando tanto la valoración de la dependencia como el reconocimiento, en su caso, del derecho efectivo.

${ }^{20}$ Atendiendo a los datos estadísticos del Imserso, se comprueba que desde junio de 2011 hasta noviembre de 2013 el número de personas con grado III de dependencia reconocido se ha reducido en 103.006, hasta situarse en 332.658 (un $23,46 \%$ menos). 
ARENAS VIRUEZ, M. (2013): “La efectividad de los principios rectores de la política social y económica de la Constitución española en el ámbito presupuestario y planificador: repercusión en la protección y atención a las personas en situación de dependencia”, en GARRIDO PÉREZ, E. (coord.), Constitución española y relaciones laborales ante el actual escenario social y económico, serie Monografías de Temas Laborales, n- 51 , Sevilla, Consejo Andaluz de Relaciones Laborales, págs. 591-602.

BARRIGA, L. A. et al. (2014): XIII Dictamen del Observatorio Estatal de la Dependencia, serie Dictámenes del Observatorio Estatal de la Dependencia, Asociación Estatal de Directoras y Gerentes de Servicios Sociales [<http://www. directoressociales.com/images/documentos/ dictamenes/XIII\%20DICTAMEN\%20del\%20 OBSERVATORIO.pdf)].

- (2013): X Dictamen del Observatorio Estatal de la Dependencia, serie Dictámenes del Observatorio Estatal de la Dependencia, Asociación Estatal de Directoras y Gerentes de Servicios Sociales [«http://www. directoressociales.com/images/ $\mathrm{x} \% 20$ dictamen\%20.pdf〉].

BELTRÁN AGUIRRE, J. L. (2008): “La atención a la dependencia: régimen legal. Interacciones entre lo social y lo sanitario", Derecho y Salud, vol. $16, \mathrm{n}^{0}$ extra 2, págs. 3-27.

COLÁS NEILA, E. (2013): "La financiación del sistema de autonomía y atención a la dependencia”, en GONZÁLEZ ORTEGA, S. (dir.), La aplicación de la Ley de Dependencia en España, Madrid, Consejo Económico y Social, págs. 99-166.

CONSEJO DE EUROPA (1998): “Recomendación no 98, 9, del Comité de Ministros a los Estados miembros relativa a la dependencia (aprobada el 18 de septiembre de 1998 , en la $641^{\circ}$ reunión de delegados de ministros)".

CONSEJO ECONÓMICO Y SOCIAL (2006): Dictamen sobre el anteproyecto de Ley de Promoción de la Autonomía Personal y Atención a las Personas en Situación de Dependencia, colección Dictámenes, no 3/2006, Madrid, Consejo Económico y Social [<http://www.ces.es/ documents/10180/18507/Dic032006)].

CORREA GÓMEZ, M.; MONTERO GRANADOS, R.; y JIMÉNEZ AGUILERA, J. D. (2011): "Valoración de inequidad en el nivel acordado de financiación de la Ley de Dependencia", en XVIII Encuentro de Economía Pública [<http://dialnet.unirioja. es/descarga/articulo/3632273.pdf〉].

DURÁN BERNARDINO, M. (2014): “La situación económica y financiera del sistema de dependencia: una insostenibilidad inducida", Revista del Centro de Estudios Financieros, ํㅡㅇㅡ.

ESPAÑA (2014): “Ley 36/2014, de 26 de diciembre, de Presupuestos Generales del Estado para el año 2015", Boletín Oficial del Estado, № 315, 30-1214, págs. 106.153-106.659 [<http://www.boe. es/buscar/doc.php?id=BOE-A-2014-13612〉].

- (2013a): “Ley 22/2013, de 23 de diciembre, de Presupuestos Generales del Estado para el año 2014", Boletín Oficial del Estado, no 309, 26-1213, págs. 104.609-105.136 [<http://www.boe. es/buscar/doc.php?id=BOE-A-2013-13616>].

- (2013b): “Real Decreto 1050/2013, de 27 de diciembre, por el que se regula el Nivel Mínimo de Protección Establecido en la Ley 39/2006, de 14 de diciembre, de Promoción de la Autonomía Personal y Atención a las Personas en Situación de Dependencia”, Boletín Oficial 
del Estado, n- 313, 31-12-13, págs. 107.120107.127 [<http://www.boe.es/buscar/doc. php?id=BOE-A-2013-13810>].

- (2013C): "Real Decreto 1051/2013, de 27 de diciembre, por el que se regulan las Prestaciones del Sistema para la Autonomía y Atención a la Dependencia, Establecidas en la Ley 39/2006, de 14 de diciembre, de Promoción de la Autonomía Personal y Atención a las Personas en Situación de Dependencia”, Boletín Oficial del Estado, $\mathrm{n}^{0}$ 313, 31-12-13, págs. 107.128107.142 [<http://www.boe.es/buscar/doc. php?id=BOE-A-2013-13811)].

- (2012a): “Ley 2/2012, de 29 de junio, de Presupuestos Generales del Estado para el año 2012", Boletín Oficial del Estado, no 156, 30-6-12, págs. 46.432-46.935 [<http://www.boe.es/buscar/ doc.php?id=BOE-A-2012-8745 $)$.

- (2012b): “Ley 17/2012, de 27 de diciembre, de Presupuestos Generales del Estado para el año 2013", Boletín Oficial del Estado, no 312, 28-1212, págs. 88.156-88.670 [<http://www.boe.es/ buscar/doc.php?id=BOE-A-2012-15651>].

- (2012c): "Real Decreto-ley 20/2012, de 13 de julio, de Medidas para Garantizar la Estabilidad Presupuestaria y de Fomento de la Competitividad", Boletín Oficial del Estado, no $168,14-7-12$, págs. 50.428-50.518 [<http://www.boe.es/diario_boe/txt. php?id=BOE-A-2012-9364>].

- (2012d): "Resolución de 13 de julio de 2012, de la Secretaría de Estado de Servicios Sociales e Igualdad, por la que se publica el Acuerdo del Consejo Territorial del Sistema para la Autonomía y Atención a la Dependencia para la Mejora del Sistema para la Autonomía y Atención a la Dependencia", Boletín Oficial del Estado, $\mathrm{n}^{0}$ 185, 3-8-12, págs. 55.65755.674 [rhttp://www.boe.es/buscar/doc. php?id=BOE-A-2012-10468>].

- (2011): “Real Decreto Ley 20/2011, de 30 de diciembre, de Medidas Urgentes en Materia Presupuestaria, Tributaria y Financiera para la Corrección del Déficit Público", Boletín Oficial del Estado, no 315 , 31-12-11, págs. 146.574146.648 [ [http://www.boe.es/buscar/doc. php?id=BOE-A-2011-20638>].

- (2010): “Real Decreto 373/2010, de 26 de marzo, por el que se determina el nivel mínimo de protección garantizado a los beneficiarios del Sistema para la Autonomía y Atención a la Dependencia para el ejercicio 2010", Boletín Oficial del Estado, nㅜ 75, 27-3-10, págs. 29.07929.081 [<http://www.boe.es/buscar/doc. php?id=BOE-A-2010-5039'].

- (2009): “Real Decreto 74/2009, de 30 de enero, por el que se determina el nivel mínimo de protección garantizado a los beneficiarios del sistema para la autonomía y atención a la dependencia para el ejercicio 2009", Boletín Oficial del Estado, no 7, 31-1-09, págs. 10.42810.430 [khttp://www.boe.es/buscar/doc. php?id=BOE-A-2009-1600〉].

- (2008a): "Real Decreto 179/2008, de 8 de febrero, por el que se modifica el Real Decreto 6/2008, de 11 de enero, sobre determinación del nivel mínimo de protección garantizado a los beneficiarios del Sistema para la Autonomía y Atención a la Dependencia en el ejercicio 2008", Boletín Oficial del Estado, nํ3, 9-2-08, págs. 7.1627.163 [<http://www.boe.es/buscar/doc. php?id=BOE-A-2008-2250〉].

- (2008b): “Resolución de 2 de diciembre de 2008 , de la Secretaría de Estado de Política Social, Familias y Atención a la Dependencia y a la Discapacidad, por la que se publica el Acuerdo del Consejo Territorial del Sistema para la Autonomía y Atención a la Dependencia, sobre determinación de la capacidad económica del beneficiario y sobre los criterios de participación de éste en las prestaciones del Sistema para la Autonomía y Atención a la Dependencia", Boletín Oficial del Estado, no 303, 17-12-08, págs. 50.72550.726 [ khttp://www.boe.es/buscar/doc. php?id=BOE-A-2008-20452〉].

- (2007a): “Real Decreto 614/2007, de 11 de mayo, sobre Nivel Mínimo de Protección del Sistema para la Autonomía y Atención a la Dependencia Garantizado por la Administración General del Estado", Boletín Oficial del Estado, $\mathrm{n}-114,12-5$ 07, págs. 20.601-20.602 [<http://www.boe.es/ buscar/doc.php?id=BOE-A-2007-9689>].

- (2007b): “Real Decreto 615/2007, de 11 de mayo, por el que se regula la Seguridad Social de los cuidadores de las Personas en Situación de Dependencia”, Boletín Oficial del Estado, no 114, 12-5-07, págs. 20.60220.605 [ [http://www.boe.es/buscar/doc. php?id=BOE-A-2007-9690>].

- (2006): “Ley 39/2006, de 14 de diciembre, de Promoción de la Autonomía Personal y Atención a las personas en situación de dependencia", Boletín Oficial del Estado, no 299, 15-12-06, págs. 44.142-44.156 [<http://www.boe.es/boe/ dias/2006/12/15/pdfs/A44142-44156.pdfs].

GONZÁLEZ ORTEGA, S. (2013): “La crisis del sistema y las medidas económicas y legales adoptadas para abordarla”, en GONZÁLEZ ORTEGA, S. (dir.), La aplicación de la Ley de Dependencia en España, Madrid, Consejo Económico y Social, págs. 5-28.

IMSERSO (s/d): Sistema de Autonomía y Financiación a la Dependencia. Estadísticas. Datos de gestión [<http://www.dependencia.imserso.es/ dependencia_01/documentacion/estadisticas/ index.htm〉].

MINISTERIO DE EMPLEO Y SEGURIDAD SOCIAL (s/d): Informe de afiliados ocupados a la Seguridad Social. Diciembre de 2012, Ministerio de Empleo y Seguridad Social.

MINISTERIO DE SANIDAD, POLITICA SOCIAL E IGUALDAD (2011): Informe del Gobierno para la Evaluación de la Ley de Promoción de la Autonomía personal y Atención a las Personas en Situación de Dependencia, Ministerio de Sanidad, Política Social e Igualdad [khttp://www. dependencia.imserso.es/dependencia_01/ documentacion/evo_doc/d/inf_eval/>].

MOLERO MARAÑÓN, M. L. (coord.) (2012): Retos para una implantación efectiva del Sistema para la Autonomía y Atención a la Dependencia, Madrid, Cinca. 
MOLINA GONZÁLEZ-PUMARIEGA, R. (2012): “LoS servicios del Sistema para la Autonomía y Atención a la Dependencia tras seis años de funcionamiento", en MOLERO MARAÑÓN, M. L. (coord.), Retos para una implantación efectiva del Sistema para la Autonomía y Atención a la Dependencia, Madrid, Cinca, págs. 105-155.

MONTSERRAT CODORNIU, J.; y MONTEJO SARRIAS, I. (2011): "El copago en la Ley de Promoción de la Autonomía Personal y Atención a las
Personas en Situación de Dependencia. Costes e impactos en las rentas de los usuarios", Papeles de Economía Española, nํㅜ 129, págs. 195-206.

VILAPLANA PRIETO, C. (2012): “Valoración económica de las medidas de mejora del Sistema de Autonomía y Atención a la Dependencia aprobadas por el Real Decreto 20/2012, de 13 de julio", Actas de la Dependencia, nํ6, págs. 35-49. 